\title{
A minor allele of the haplotype located in the $19 q 13$ loci is associated with a decreased risk of hyper-LDL- cholesterolemia, and a balanced diet and high protein intake can reduce the risk
}

\author{
Sunmin Park ${ }^{*}$ and Suna Kang
}

\begin{abstract}
Background: Although the human chromosome 19q13 loci are reported to be associated with hyper-LDLcholesterolemia, the haplotype of single nucleotide polymorphism (SNP) has not been studied. Therefore, the association of the haplotype in 19q13 loci with hyper-LDL-cholesterolemia was determined and their interactions with lifestyles and nutrient intakes were evaluated in 28,445 Koreans aged $>40$ years.
\end{abstract}

Methods: SNPs were selected from 19q13 loci that had an association with hyper-LDL-cholesterolemia with the adjustment of confounders (age, gender, area of residence, and body mass index). Haplotype was constructed from the selected SNPs. An adjusted odds ratio of the haplotype for hyper-LDL-cholesterolemia and the interaction between haplotype and lifestyles was analyzed after adjusting for covariates.

Results: Hyper-LDL-cholesterolemia had an association with apolipoprotein E (APOE)_rs7259620, translocase of outer mitochondrial membrane 40(TOMM40)_rs157581, poliovirus receptor-related 2(PVRL2)_rs403155, exocyst complex component 3-like 2(EXOC3L2)_rs10406604 and CD3e molecule-associated protein (CD3EAP)_rs3212986 in 19q13. The haplotype of these SNPs had a negative association with hyper-total-cholesterolemia and hyper-LDLcholesterolemia by 0.669 and 0.684 times, respectively, after adjusting for covariates. The incidence of cardiovascular diseases, especially myocardial infarction, had a negative association with the minor alleles. The balanced diet pattern (BD) and protein intake had a significant interaction with the haplotype: the major-allele of the haplotype exhibited a positive association with hyper-LDL-cholesterolemia, compared to the minor allele, only when combined with a high intake of BD. The participants with the minor allele exhibited a lower hyper-LDLcholesterolemia risk compared to those with the major allele only with high protein intake.

Conclusion: The minor allele of haplotype located in 19q13 loci protected against hyper-LDL-cholesterolemia, especially with $\mathrm{BD}$ and high protein intake. The minor allele also had a negative association with myocardial infarction events.

Keywords: Dyslipidemia, Low-density lipoprotein, Apolipoprotein E, 19q13 loci, Protein

\footnotetext{
* Correspondence: smpark@hoseo.edu

Department of Food and Nutrition, Obesity/Diabetes Research Center, Hoseo University, 165 Sechul-Ri, BaeBang-Yup, Asan-Si, ChungNam-Do 31499, South Korea
} 


\section{Introduction}

The mortality from ischemic heart diseases has increased rapidly, possibly due to increased dyslipidemia [1]. The prevalence of hypercholesterolemia $(\geq 240 \mathrm{mg} / \mathrm{dL}$ total cholesterol or taking cholesterol-lowering medication) in 1998, 2012, and 2016 has increased continuously from $7.2,12.6$, and $19 \%$, and from $8.4,14.9$, and $20 \%$, respectively, in Korean men and women aged $\geq 30[1,2]$. Although the prevalence of dyslipidemia has increased, the awareness and treatment of dyslipidemia are relatively lower than for type 2 diabetes and hypertension [3]. In 2014, the Korean National Health Insurance changed the diagnostic criteria for dyslipidemia from a total cholesterol basis to a low-density lipoprotein cholesterol (LDL) basis because lowering the serum LDL concentration is the primary objective of dyslipidemia therapy [4]. In 2018, people with an LDL concentration of $\geq 160 \mathrm{mg} /$ $\mathrm{dL}$ with one or more major risk factors were recommended to administer cholesterol-lowering medication and lifestyle modification [5]. The risk factors include middle-age ( $>45$ years for men and $>55$ years for women), family history of premature coronary artery disease, hypertension, smoking, and hypo-high-density lipoprotein (HDL)-cholesterolemia. Elevated serum LDL concentrations need to be prevented by controlling the modifiable risk factors.

Although the genetic factors are unmodifiable, their effects can be modulated efficiently by environmental and lifestyle factors because genetic factors interact with lifestyle and environmental risk factors. Dietary patterns are one of the crucial factors for managing dyslipidemia, including hyper-LDL-cholesterolemia. The Mediterranean diet has been reported to be negatively associated with the serum LDL cholesterol concentrations in both randomized clinical trials and cross-sectional studies [6, 7]. Moreover, the Dietary Approaches to Stop Hypertension diet was reported to be negatively associated with the serum LDL concentrations [8]. Furthermore, a high-snack diet consisting largely of fried chips, cookies, sweets, ice cream, and liquid beverages is positively associated with the serum total cholesterol, LDL, and triglyceride concentrations [9]. A traditional healthy dietary pattern was reported to have an association with hyper- HDLcholesterolemia concentrations in Korean men [10].

Cholesterol is taken up into the liver by chylomicrons and very-low-density lipoprotein (VLDL) remnants, where they are recognized by the apolipoprotein $\mathrm{E}$ $(A P O E)$ receptor [11]. When large amounts of cholesterol are stored in the liver due to a high cholesterol intake and synthesis, the LDL receptor $(L D L R)$ is downregulated by lysosomal degradation after $L D L R$ binds to the proprotein convertase subtilisin-kexin type 9 (PCSK9) [12]. The serum LDL concentrations are affected by $L D L R, P C S K 9$, and $A P O E$ with the respective genes located in chromosomes 19, 1, and 19, respectively. Among the loci, 19q13 includes important genes related to cholesterol metabolism, including $L D L R$, $A P O E, A P O C 1$, and translocase of outer-mitochondrial membrane 40 (TOMM40) [13, 14]. These genes are involved in LDL uptake into the cells to lower the serum LDL concentrations utilizing the triglyceride in VLDL to modulate the serum LDL concentrations. Furthermore, peroxisome proliferator-activated receptor- $\gamma$, which is involved in lipid utilization in the liver, is associated with the expression of the genes in the $19 q 13$ loci [13]. In the Finnish cohort, the $19 \mathrm{q} 13.31$ and $19 \mathrm{q} 13.32$ loci exhibited genome-wide significance for serum LDL concentration, but the 1p32 loci did not [15]. On the other hand, the loci were reported to be associated with TG and HDL in non-Hispanic Caucasians and Africans [16]. These studies indicate that the genetic variants in 19q13.32 play an important role in the lipid metabolism, including hyperLDL-cholesterolemia.

Dietary patterns do not uniformly affect dyslipidemia among all people because of the genetic impacts. No studies have investigated the interaction between genetic and environmental risk factors or how the results can be applied to people at risk of hyper-LDL-cholesterolemia for personalized nutrition. The current study aimed to determine if genetic variants located in 19q13 have a strong association with hyper-LDL-cholesterolemia and the incidence of cardiovascular diseases, including myocardial infarction and stroke. The interaction of haplotypes with lifestyles and nutrient intakes was also investigated to modulate hyper-LDL-cholesterolemia. These were examined in 28,445 Korean middle-aged and elderly participants in a large hospital-based urban cohort.

\section{Materials and methods}

\section{Baseline characteristics of participants}

In this study, 28,445 adults, aged 40 to 69 years, consisting of 10,261 men and 18,184 women, participated. The study procedures were compliant with the Declaration of Helsinki, and they followed the ethical standards of the responsible committee, including the Institutional Review Board of the Korean National Institute of Health for the KoGES (KBP-2015-055) and Hoseo University (1041231-150,811-HR-034-01). All participants provided written informed consent. The exclusion criteria in the participants were secondary obesity with Cushing's disease, hypothyroidism, severely debilitating disease, undergoing anti-obesity treatment or weight loss for the past 6 months [17].

The baseline characteristics of the participants were collected from the survey, such as age, gender, education, income, and the status of smoking, drinking, and physical exercise. The daily physical activity was 
estimated by summing the multiplication of each activity level by the duration of physical activity at each activity level. The estimated daily physical activities were divided into low, medium, and high activity. The usual alcohol intake was determined by multiplying the average amount of alcohol consumed and the frequencies of alcohol drinking on each occasion. The daily alcohol consumption was divided into light drinking $(<1 \mathrm{~g})$, moderate drinking (1-10 g), and heavy drinking (>10 g). Daily coffee consumption was estimated in the same manner as the daily alcohol consumption. Non-smoking was defined as having smoked $<10$ packs during their entire lifetime, while former smokers had not smoked for the last 6 months before the survey. A previous diagnosis of myocardial infarction and stroke events by a medical doctor was asked.

\section{Anthropometric and biochemical measurements}

In the anthropometric measurements, the body weight, height, and waist and hip circumferences were measured three times as specified in a manual explained in previous studies $[17,18]$. The body mass index was calculated by weight $(\mathrm{kg}) /$ height $^{2}\left(\mathrm{~m}^{2}\right)$ using the average values. In biochemical measurements of blood from the participants in an overnight-fasted state, the serum triglyceride, total cholesterol, and HDL concentrations, and plasma glucose concentrations were determined using a Hitachi 7600 Automatic Analyzer (Hitachi, Tokyo, Japan). The LDL was calculated using the Friedwald equation (total cholesterol - HDL - triglyceride/5 in the serum). Because Friedwald equation could not estimate serum LDL concentrations in serum triglyceride concentrations $>400 \mathrm{mg} / \mathrm{dL}$ [19], subjects with $>400$ $\mathrm{mg} / \mathrm{dL}$ were excluded.

\section{Nutrient intake assessment}

The usual food intake of each participant was estimated using a semi-quantitative food frequency questionnaire (SQFFQ) that was validated by the three-day foodrecords for three seasons in a previous study [19]. The SQFFQ included 103 food items usually included in Korean meals. The portion sizes of each food item were categorized as half, one, and double of a single portion size to be shown as pictures. The amounts of each food item intake were measured by multiplying the frequencies of each food intake by its portion size [18]. The nutrient intakes, including energy, carbohydrate, protein, and fat, were calculated from the food intakes collected by the SQFFQ using Can-Pro 2.0 software designed for calculating the nutrient intake from the food intake. The nutrient program was developed by the Korean Nutrition Society (Seoul, Korea).

\section{Genotyping and quality control}

Genotyping was conducted from the DNA extracted from the peripheral white blood cells of the subjects using the Axiom Biobank Plus Genotyping Array, KNIHv1.1 (Affymetrix, Santa Clara, CA, USA) [17]. Single nucleotide polymorphisms (SNPs) were excluded because they did not meet the criteria, including no gender bias, genotyping accuracy (>98\%), and low missing genotype call rate $(<4 \%)$ for quality control and quality assurance.

The genetic variants associated with the risk of hyperLDL-cholesterolemia were examined through a genomewide association study (GWAS), and 43 genetic variants were selected at chromosome 19 at $P<0.0001$. According to Bayesian-Robust Linear Modeling of the Mahalanobis distance classifier genotype-calling algorithm, the discordance of batches with different batch sizes was approximately $2 \%$. The gene names of the selected genetic variants were found using scandb.org, and SNPs related to LDL metabolism, including $A P O E$ in chromosome 19, were chosen. In the haplotype generation, the genetic variants included satisfied the following criteria: minor allele frequency $(\mathrm{MAF})>0.01$, SNP missing rate $<0.1$, and Hardy-Weinberg equilibrium (HWE; $P>0.05$ ). Linkage disequilibrium (LD) analyses were performed in the selected genetic variants from the same chromosome using Haploview 4.2 in PLINK version 1.90b (http:// pngu.mgh.harvard.edu/ purcell/plink). The SNPs with strong LD ( $\left.\mathrm{D}^{\prime}>0.4\right)$ in the pairwise SNPs were excluded because they provided similar information to hyperLDL-cholesterolemia risk.

\section{Genetic variants to have an association with hyper-LDL- cholesterolemia risk in Koreans}

The participants were categorized into low-LDL and High-LDL groups by $\geq 160 \mathrm{mg} / \mathrm{dL}$ or taking cholesterollowering medication (case) and serum LDL concentrations $<160 \mathrm{mg} / \mathrm{dL}$ (control), regardless of age and gender based on the "Korean guidelines for the management of dyslipidemia in 2018" from the Korean Society of Lipid and Atherosclerosis and the Korean Association of Internal Medicine since the participants were $>40$ years old. GWAS was conducted to explore the SNPs involved in hyper-LDLcholesterolemia with the case and control groups with adjusting for the covariates of age, gender, residential area, and body mass index (BMI) using PLINK software. The gene names of the SNPs were identified using the SCAN database (http://scandb. org/newinterface/). The important SNPs that influenced the risk of hyper-LDL-cholesterolemia were selected, and their LD plot was generated using Haploview using PLINK version 1.90b software. 


\section{Haplotype analysis}

A haplotype is more powerful than a single SNP based analysis. SNPs adjacent to each other on the same chromosome were selected for haplotype analysis. Haplotype analysis was conducted from the SNPs selected from GWAS using PLINK software. Each SNP selected for the haplotype was given a score of 0,1 , or 2 according to the major allele, heterozygote allele, and minor allele, respectively. Haplotype scores were calculated by adding the scores of each SNP included in the haplotype. Haplotype alleles were categorized into three categories $(0,1-2$, and 3-4) by tertiles for the major-allele (Major), heterozygoteallele (Heterozygote), and minor-allele (Minor) groups of the haplotype, respectively.

\section{Interactions between the haplotype and lifestyles on hyper-LDL-cholesterolemia}

The daily nutrient intakes, including energy, protein, fat, and carbohydrate, as well as daily coffee and alcohol intake, were divided into high- and low-intake groups. The percentage of daily energy requirements consumed was calculated by dividing the daily energy intakes by the estimated dietary energy requirement of Koreans in 2016 [20]; $2400 \mathrm{kcal}$ for < 50-year-old men; $2200 \mathrm{kcal}$ for $\geq 50$ year-old men; $1900 \mathrm{kcal}$ for < 50 -year-old women; and $1800 \mathrm{kcal}$ for $\geq 50$-year-old women. The interactions between the lifestyles and haplotypes on hyper-LDLcholesterolemia were analyzed using a two-way analysis of variance (ANOVA) including main effect terms and the interaction terms of haplotypes and lifestyles with adjusting for covariates. The covariates were the residential area, age, gender, BMI, alcohol and coffee intake, smoking status, menopause, and total activity.

\section{Statistical analysis}

Statistical analysis was conducted using SAS version 9.4 (SAS Institutes, Cary, NC, USA). A two-sample t-test was used to analyze the statistical significance of the continuous variables, such as age, BMI, waist circumference, and glucose and lipid profiles, in the blood between the Low- and High-LDL groups. Their means and standard deviations were also calculated. The frequency distributions were analyzed in the classification variables (haplotype alleles, gender, myocardial infarction, and stroke) using a chi-squared test. Significant intergroup differences of the haplotype alleles were determined by one-way ANOVA by adjusting for different sets of covariates. Multiple comparisons of the groups were performed using a Bonferroni test.

The associations between the haplotype and the hyper-LDL-cholesterolemia risk were assessed using multiple logistic regression analysis, adjusting for different sets of covariates. The adjusted odds ratios (ORs) plus $95 \%$ confidence intervals $(\mathrm{CI})$ were calculated using the major haplotype allele as a reference in the two models by multiple logistic regression analysis with an adjustment for two sets of covariates. The association of the genetic effect with hyper-LDL-cholesterolemia, according to the haplotype, was explored after offsetting all the potential effectors of hyper-LDL-cholesterolemia as covariates. The covariates of model 1 were the basic confounders, and those of model 2 included all potential confounders to influence the serum LDL concentrations. The covariates in model 1 were age, gender, residential area, BMI and energy intake, and the parameters in the model 1 plus energy, coffee, and alcohol intakes, smoking status, physical activity, and serum total cholesterol concentrations belonged to model 2 as covariates.

The interactions between the SNPs and lifestyles were determined by allocating the participants to low- and highintake groups by the 75th percentiles. The interactions between the haplotype and lifestyles were analyzed by twoway ANOVA including an interaction term with covariates. Their effects on the hyper-LDL-cholesterolemia risk were assessed by multiple logistic analysis after adjusting for the covariates in the low- and high-groups. $P$ values $<0.05$ were considered significant.

\section{Results \\ General characteristics of the participants according to hyper-LDL-cholesterolemia}

The participants were older in the High-LDL group than the Low-LDL group in both genders. Subjects in the High-LDL group $(\geq 160 \mathrm{mg} / \mathrm{dL}$ serum LDL cholesterol levels) were more likely to be obese (higher BMI and waist circumferences) than those in the Low-LDL group $(<160$ $\mathrm{mg} / \mathrm{dL}$ serum LDL cholesterol levels) in both genders (Table 1). Serum total cholesterol, HDL, LDL, and triglyceride concentrations in the High-LDL group were much higher than the Low-LDL group in both genders (Table 1 ). Therefore, both genders were combined for searching genetic variants to influence serum LDL concentrations. However, the incidence of cardiovascular diseases combined myocardial infarction and stroke was significantly higher in the High-LDL group than the Low-LDL group in women but not in men (Table 1). However, each event of myocardial infarction and stroke did not show significant differences between the Low-LDL and High-HDL groups in both genders. In the High-LDL group, cardiovascular disease incidence was higher by about 2.5 times than the Low-LDL group. When the exploration of genetic variants is conducted for cardiovascular diseases, gender differences need to be considered.

\section{Selection of the genetic variants associated with hyper- LDL-cholesterolemia risk}

In the $19 \mathrm{q} 13$ loci, hyper-LDL-cholesterolemia was associated with $A P O E$ rs7259620, poliovirus receptor-related 
Table 1 General characteristics of the study population according to serum LDL concentration

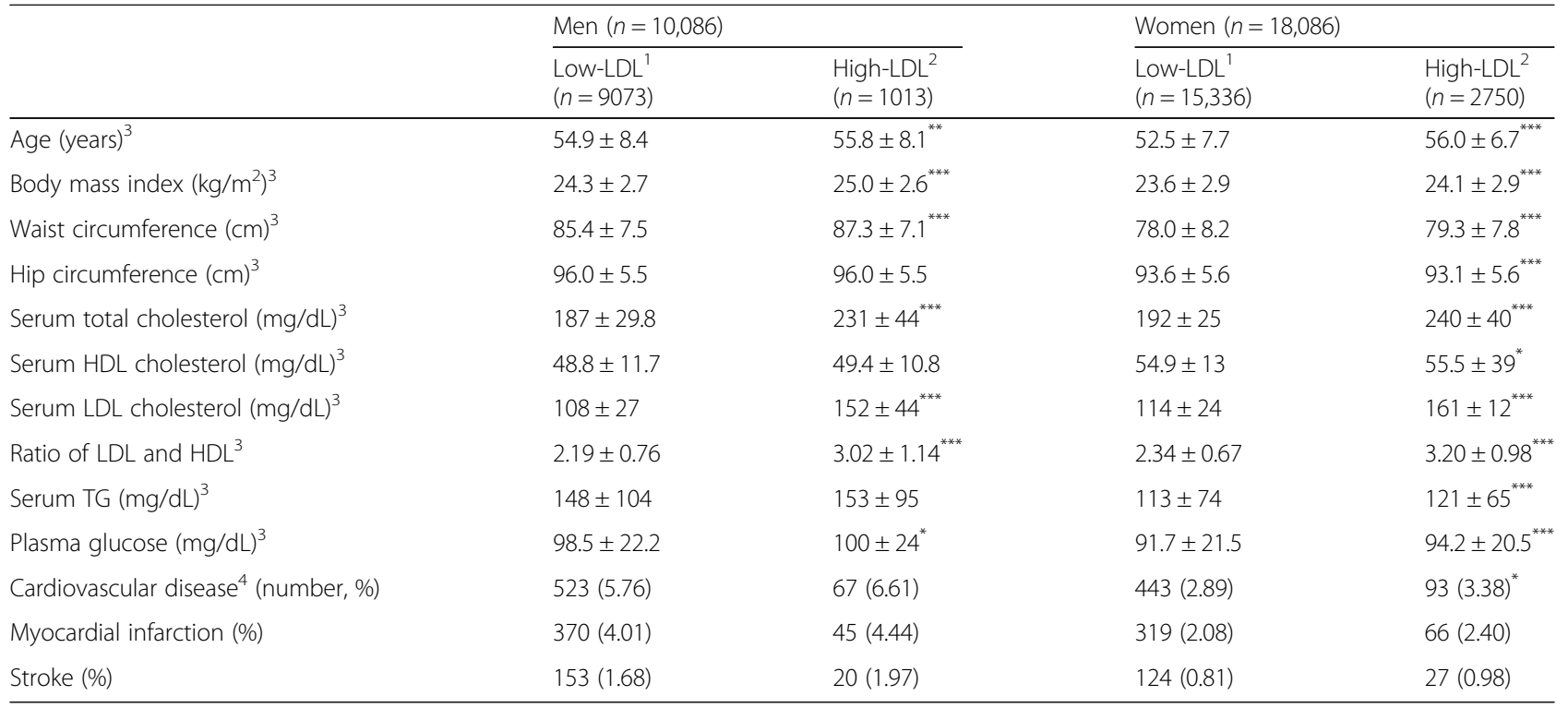

The values represent means \pm standard deviations or number of the subjects (percentage of each group). ${ }^{1}$ Low serum LDL cholesterol concentrations $<160$ mg/ $\mathrm{dl}^{2}{ }^{2}$ High serum LDL cholesterol concentrations $\geq 160 \mathrm{mg} / \mathrm{dl}$ and/or taking lipid-lowering medicine;

Significantly different from Low-LDL group in men and women at ${ }^{*} P<0.05 ;{ }^{* *} P<0.01 ;{ }^{* * *} P<0.001$

2 (PVRL2) rs403155 TOMM40 rs157581, exocyst complex component 3-like 2 (EXOC3L2) rs10406604 and CD3e molecule associated protein (CD3EAP) rs3212986 and these SNPs were located from 45,363,299 to 45,912, 736 in chromosome 19 (Table 2). Adjusted ORs of these SNPs were $0-1$ and the $P$ values were between 1.64E-4 and 3.79E-19 (Table 2). The SNPs satisfied the HWE criteria $(P>0.05)$. LD of 5 SNPs is presented in Fig. 1. The lighter color in the haploview indicated a lower correlation between the adjacent SNPs in the haplotype. The numbers in the triangles represented the D' "100 between the adjacent SNPs [21]. Low correlations between adjacent SNPs $\left(D^{\prime}<0.2\right)$ suggested that the SNPs met the requirements to be included in the haplotype analysis. Although EXOC3L2 rs10406604 and CD3EAP rs3212986 did not meet the GWAS statistical significance $(P$ value $<5.0 \mathrm{E}-8)$, they showed statistical significance (1.94E-4 and 8.58E-05) and the haplotype including 2 SNPs provided a better model to show a more significant association with lipid profiles and cardiovascular diseases risk. The haplotype including 5 SNPs were used for further analysis. The logistic estimated coefficients and ORs of the haplotype including 3 SNPs were provided in Supplemental Table 1.

\section{Adjusted means of participant characteristics according to the haplotype generated from hyper-LDL- cholesterolemia}

BMI and waist circumferences were not changed by haplotype but they were greater in participants in the High-LDL group than those in the Low-LDL group in two-way ANOVA $(P<0.001$; Table 3$)$. Serum total cholesterol, LDL, and triglyceride concentrations were lower in the carriers of the minor allele than those of the major allele $(\mathrm{P}<0.001$; Table 3$)$ and they were higher in the High-LDL group in the than the Low-LDL group $(\mathrm{P}<0.001)$. The ratio of LDL to HDL was influenced by the haplotype and LDL groups $(\mathrm{P}<0.001)$ although

Table 2 The characteristics of the three genetic variants used for haplotype located in the 19q13 loci

\begin{tabular}{llllllllll}
\hline SNP & Position & Mi & Ma & OR & P value for OR & MAF & HWE_P & Gene & Location \\
\hline rs7259620 & $45,407,788$ & A & G & 0.834 & $3.79 E-19$ & 0.284 & 0.737 & APOE & near-gene-5 \\
rs403155 & $45,363,299$ & T & C & 0.789 & $3.30 E-15$ & 0.107 & 0.475 & PVRL2 & intron \\
rs157581 & $45,395,714$ & C & T & 0.911 & $2.64 E-19$ & 0.236 & 0.250 & TOMM40 & coding-synonymous \\
rs10406604 & $45,723,986$ & A & G & 0.917 & $8.58 E-05$ & 0.221 & 0.094 & EXOC3L2 & intron \\
rs3212986 & $45,912,736$ & A & C & 0.926 & $1.64 E-04$ & 0.266 & 0.976 & CD3EAP & missense \\
\hline
\end{tabular}

APOE Apolipoprotein E, PVRL2 Poliovirus receptor-related 2 (nectin-2 and CD112), TOMM40 Translocase of outer mitochondrial membrane 40 homolog, EXOC3L2 Exocyst complex component 3 Like 2, CD3EAP CD3e molecule associated protein, CHR Chromosome, SNP Single nucleotide polymorphism, Mi Minor allele, Ma Major allele, $O R$ Odds ratios (OR) for serum LDL concentration in the reference of the major allele; $P$ value for OR adjusted for age, gender, residence area, body mass index, and energy intake, MAF Minor allele frequency, $H W E_{-} P P$ value for Hardy-Weinberg equilibrium 


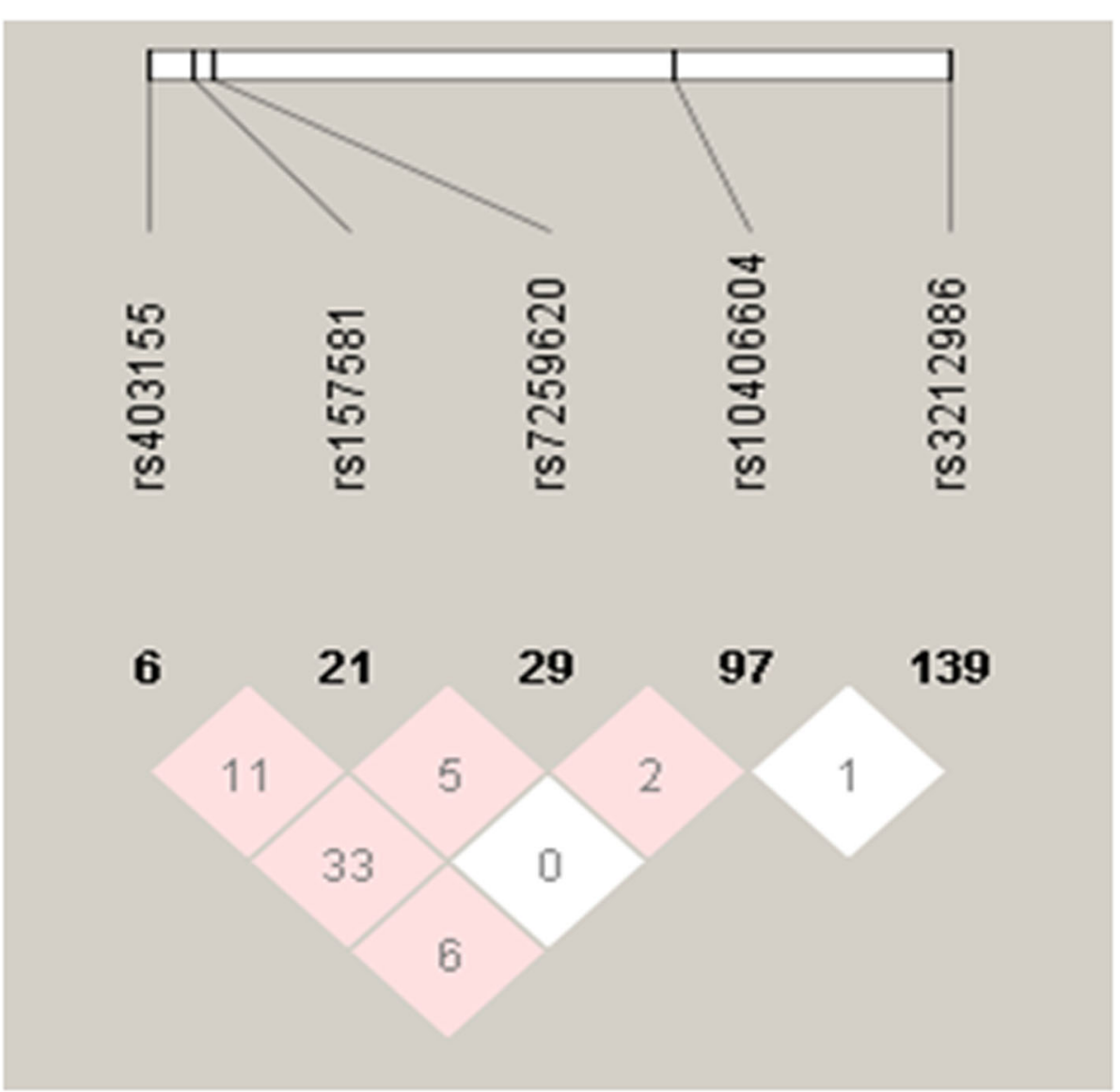

Fig. 1 Haploview in the 19q13 loci. Haploview of the 19q13 loci. Each value in the colored rectangle represents the D' between a pair of SNPS and the range of the value $0-100$. The lighter color in the haploview indicates a lower correlation between the adjacent SNPs in the haplotype. The numbers in the triangles represent the correlation coefficient between the adjacent SNPS

Table 3 Adjusted means in obesity status and serum lipid profiles of participants with major-, heterozygote-, and minor-alleles of the haplotype ${ }^{1}$ according to serum LDL cholesterol concentrations

\begin{tabular}{|c|c|c|c|c|c|c|}
\hline & \multicolumn{3}{|l|}{ Low-LDL ${ }^{2}$} & \multicolumn{3}{|l|}{ High-LDL $^{3}$} \\
\hline & $\begin{array}{l}\text { Major }^{4} \\
(n=10,950)\end{array}$ & $\begin{array}{l}\text { Heterozygote } \\
(n=10,728)\end{array}$ & $\begin{array}{l}\text { Minor } \\
(n=2731)\end{array}$ & $\begin{array}{l}\text { Major } \\
(n=1782)\end{array}$ & $\begin{array}{l}\text { Heterozygote } \\
(n=1680)\end{array}$ & $\begin{array}{l}\text { Minor } \\
(n=301)\end{array}$ \\
\hline BMI $\left(\mathrm{kg} / \mathrm{m}^{2}\right)$ & $23.8 \pm 2.8^{b}$ & $23.9 \pm 2.9^{b}$ & $23.9 \pm 2.8^{b}$ & $24.3 \pm 2.9^{a}$ & $24.4 \pm 2.8^{a}$ & $24.2 \pm 2.6^{b+++}$ \\
\hline$W C(\mathrm{~cm})$ & $80.6 \pm 8.7^{b}$ & $80.8 \pm 8.8^{b}$ & $80.7 \pm 8.6^{b}$ & $81.9 \pm 8.3^{\mathrm{a}}$ & $81.8 \pm 8.2^{\mathrm{a}}$ & $81.3 \pm 7.4^{\mathrm{b}+++}$ \\
\hline Total cholesterol (mg/dl) & $191 \pm 29^{b}$ & $190 \pm 29^{b}$ & $186 \pm 30^{c}$ & $238 \pm 40^{a}$ & $239 \pm 41^{a}$ & $235 \pm 48^{\mathrm{a}^{* * * *++}}$ \\
\hline LDL (mg/dl) & $114 \pm 25^{c}$ & $112 \pm 26^{d}$ & $106 \pm 26^{\mathrm{e}}$ & $159 \pm 39^{a}$ & $158 \pm 41^{a}$ & $153 \pm 47^{b^{* * *+++}}$ \\
\hline $\mathrm{HDL}(\mathrm{mg} / \mathrm{dl})$ & $52.6 \pm 13$ & $52.8 \pm 13$ & $53.3 \pm 13$ & $53.2 \pm 12$ & $53.1 \pm 11$ & $53.2 \pm 12$ \\
\hline TG $(\mathrm{mg} / \mathrm{dl})$ & $123 \pm 88^{\mathrm{a}}$ & $125 \pm 87^{\mathrm{ab}}$ & $132 \pm 91^{c}$ & $131 \pm 77^{\mathrm{bc}}$ & $136 \pm 71^{b c}$ & $143 \pm 83^{\mathrm{c}^{* *+++}}$ \\
\hline Ratio of LDL/ HDL & $2.34 \pm 0.70^{b}$ & $2.31 \pm 0.72^{b}$ & $2.22 \pm 0.70^{c}$ & $2.65 \pm 1.04^{\mathrm{a}}$ & $2.62 \pm 1.02^{\mathrm{a}}$ & $2.56 \pm 1.65^{\mathrm{a}^{* * *}+-}$ \\
\hline
\end{tabular}

${ }^{1}$ Haplotypes of APOE, PVRL2, TOMM40, EXOC3L2, and CD3EAP in the $19 \mathrm{q} 13$ loci; ${ }^{2}$ Low serum LDL cholesterol $<160 \mathrm{mg} / \mathrm{dl}$ and ${ }^{3}$ High serum LDL cholesterol $\geq 160$ $\mathrm{mg} / \mathrm{dl} ;{ }^{4}$ Major, heterozygotes, and minor alleles of each SNP were scored 0,1 , and 2, respectively, and the scores of the SNPs in the haplotype were added. They were divided into 3 categories (Major, Heterozygote, and Minor haplotype groups) by 0,1-2, and 3-4, respectively. Adjusted for age, gender, residence area, body mass index (BMI), and energy intake. Values represent adjusted means \pm standard deviations

Significantly different from major allele group at ${ }^{*} P<0.05 ;{ }^{* *} P<0.01 ;{ }^{* * *} P<0.001$. Significantly different by LDL status ${ }^{+} P<0.05 ;{ }^{++} P<0.01 ;{ }^{+++} P<0.001$

a,b,c Means without a common letter differ in the same row by Bonferroni test at $P<0.05$ 
serum HDL concentrations were not different according to LDL groups and haplotype (Table 3 ).

\section{A negative association of the haplotype associated with hyper-LDL-cholesterolemia}

Adjusted ORs for hyper-total-cholesterolemia and hyper-LDL-cholesterolemia showed a negative association with the haplotype with the adjustment for model 1 covariates including BMI, residence area, gender, age, and energy intake and model 2 covariates including the parameters of model 1 plus smoking status, physical activity, menopause, serum total cholesterol levels and intake of energy, fat percent, carbohydrates percent, cholesterol, coffee, and alcohol (model 2; Table 4). Since there were no significant interactions with gender $(P=$ $0.638)$, age $(P=0.120)$ and BMI $(P=0.989)$, the genetic effect on lipid profiles were analyzed in model 1 and 2 without separation by age, gender, or BMI.

The adjusted ORs for hyper-total-cholesterolemia were lower in the carriers with minor alleles of the haplotype by 0.669 times in model 2 than in those with its major alleles (Table 4). Adjusted ORs for hyper-LDLcholesterolemia in the carriers with minor alleles of the haplotype were lower by 0.675 and 0.684 times in models 1 and 2, respectively, compared to those with its major allele (Table 4). Moreover, hypo-HDLcholesterolemia had a negative association with the haplotype in model 2 , but not model 1 , indicating that hypo-HDL-cholesterolemia risk was higher in the major allele of the haplotype, in comparison to its minor allele. The ratio of serum LDL/HDL was also significantly lower by 0.770 times in model 2 , indicating that the participants carrying the minor allele of the haplotype exhibited lower ratios than those carrying the major allele (Table 4). However, serum triglyceride concentrations were the opposite of serum LDL concentrations. Participants with minor alleles had a hyper-triglyceridemia risk compared to those with the major allele.

Interestingly, the risk of cardiovascular diseases was lowered by 0.746 times in the minor allele of the haplotype than its major allele only in model 2 (Table 4). Among cardiovascular diseases, the events of myocardial infarction, but not a stroke, had a negative association with haplotypes in model 2.

\section{Interaction of haplotype and lifestyle in hyper-LDL- cholesterolemia}

A balanced diet pattern (BD) explained $44.4 \%$ of Korean diet patterns. The BD pattern had a positive association with consuming beans, potatoes, kimchi, green and

Table 4 The estimated logistic regression coefficients ( $\beta^{\prime}$ s), adjusted odds ratios and 95\% confidence intervals for the risk of lipid profiles according to the alleles of the haplotype ${ }^{a}$ according to serum LDL cholesterol concentrations after covariate adjustments

\begin{tabular}{|c|c|c|c|c|c|}
\hline & \multicolumn{3}{|l|}{ Model 1} & \multicolumn{2}{|l|}{ Model 2} \\
\hline & $\begin{array}{l}\text { Major } \\
(n=1782)\end{array}$ & $\begin{array}{l}\text { Heterozygote } \\
(n=1680)\end{array}$ & $\begin{array}{l}\text { Minor } \\
(n=301)\end{array}$ & $\begin{array}{l}\text { Heterozygote } \\
(n=1680)\end{array}$ & $\begin{array}{l}\text { Minor } \\
(n=301)\end{array}$ \\
\hline $\begin{array}{l}\text { Total cholesterol } \\
(\mathrm{mg} / \mathrm{dl})\end{array}$ & 1 & $\begin{array}{l}0.084,0.940 \\
(0.893 \sim 0.988)\end{array}$ & $\begin{array}{l}-0.234^{* * *}, 0.683 \\
(0.629 \sim 0.742)\end{array}$ & $\begin{array}{l}0.082,0.928 \\
(0.881 \sim 0.978)\end{array}$ & $\begin{array}{l}-0.242^{* * *}, 0.669 \\
(0.614 \sim 0.730)\end{array}$ \\
\hline $\begin{array}{l}\mathrm{LDL}^{\mathrm{c}} \\
(\mathrm{mg} / \mathrm{dl})\end{array}$ & 1 & $\begin{array}{l}0.098,0.898 \\
(0.853 \sim 0.946)\end{array}$ & $\begin{array}{l}-0.249^{* * * *}, 0.675 \\
(0.592 \sim 0.769)\end{array}$ & $\begin{array}{l}0.089,0.956 \\
(0.842 \sim 0.975)\end{array}$ & $\begin{array}{l}-0.237^{* * *}, 0.684 \\
(0.598 \sim 0.782)\end{array}$ \\
\hline $\begin{array}{l}\mathrm{HDL}^{\mathrm{d}} \\
(\mathrm{mg} / \mathrm{dl})\end{array}$ & 1 & $\begin{array}{l}0.006,0.969 \\
(0.917 \sim 1.023)\end{array}$ & $\begin{array}{l}-0.056,0.908 \\
(0.837 \sim 0.999)\end{array}$ & $\begin{array}{l}0.031,0.966 \\
(0.912 \sim 1.023)\end{array}$ & $\begin{array}{l}-0.110^{* * *}, 0.845 \\
(0.769 \sim 0.928)\end{array}$ \\
\hline $\begin{array}{l}\text { Triglyceride } \\
(\mathrm{mg} / \mathrm{dl})\end{array}$ & 1 & $\begin{array}{l}-0.026,1.088 \\
(1.028 \sim 1.151)\end{array}$ & $\begin{array}{l}0.125^{* * *}, 1.243 \\
(1.138 \sim 1.358)\end{array}$ & $\begin{array}{l}-0.048,1.110 \\
(1.046 \sim 1.177)\end{array}$ & $\begin{array}{l}0.188^{* * *}, 1.387 \\
(1.265 \sim 1.522)\end{array}$ \\
\hline Ratio of $\mathrm{LD}$ and $\mathrm{HDL}^{f}$ & 1 & $\begin{array}{l}0.072^{* *}, 0.905 \\
(0.854 \sim 0.959)\end{array}$ & $\begin{array}{l}-0.244^{* * *}, 0.667 \\
(0.604 \sim 0.737)\end{array}$ & $\begin{array}{l}0.041,0.916 \\
(0.876 \sim 1.000)\end{array}$ & $\begin{array}{l}-0.160^{* * *}, 0.770 \\
(0.687 \sim 0.864)\end{array}$ \\
\hline Cardiovascular diseases & 1 & $\begin{array}{l}0.050,1.018 \\
(0.895-1.158)\end{array}$ & $\begin{array}{l}-0.096,0.858 \\
(0.686-1.073)\end{array}$ & $\begin{array}{l}0.076,0.968 \\
(0.846-1.108)\end{array}$ & $\begin{array}{l}-0.184^{*}, 0.746 \\
(0.592-0.941)\end{array}$ \\
\hline Myocardial infarction & 1 & $\begin{array}{l}0.067,0.987 \\
(0.783-1.245)\end{array}$ & $\begin{array}{l}-0.121,0.922 \\
(0.624-1.363)\end{array}$ & $\begin{array}{l}0.086,0.965 \\
(0.825-1.128)\end{array}$ & $\begin{array}{l}-0.208^{*}, 0.719 \\
(0.549-0.943)\end{array}$ \\
\hline Stroke & 1 & $\begin{array}{l}0.002,1.024 \\
(0.882-1.189)\end{array}$ & $\begin{array}{l}-0.033,0.831 \\
(0.639-1.082)\end{array}$ & $\begin{array}{l}0.032,0.961 \\
(0.756-1.221)\end{array}$ & $\begin{array}{l}-0.105,0.838 \\
(0.559-1.256)\end{array}$ \\
\hline
\end{tabular}

Values represent odds ratios and $95 \%$ confidence intervals

a Haplotypes of APOE, PVRL2, TOMM40, EXOC3L2, and CD3EAP in the 19q13 loci were generated by PLINK and it was divided into 3 categories (Major, Heterozygote and Minor haplotype groups) by the alleles (0,1-2, and 3-4). The Major haplotype was the reference for both model 1 and model 2

The cutoff points for dividing the values of each parameter into 2 groups were as follows: the control group included $<200 \mathrm{mg} / \mathrm{dL}$ for serum total cholesterol concentrations ${ }^{\mathrm{b}},<160 \mathrm{mg} / \mathrm{dL}$ for serum LDL concentrations (LDL) ${ }^{\mathrm{c}}, \geq 40 \mathrm{mg} / \mathrm{dL}$ for men and $\geq 50 \mathrm{mg} / \mathrm{dL}$ for women in serum HDL concentrations (HDL) ${ }^{\mathrm{d}},<150 \mathrm{mg} /$ $\mathrm{dL}$ for serum triglyceride concentrations ${ }^{\mathrm{e}}$ and 2.85 for the ratio of serum LDL concentrations to serum $\mathrm{HDL}$ concentrations ${ }^{\mathrm{f}}$

Model 1: adjusted for age, gender, residence area, body mass index, and energy intake

Model 2: adjusted for the parameter in model 1 plus smoking, coffee, alcohol, physical activity, percent of fat and carbohydrate intakes, menopause, and serum total cholesterol concentrations

" Significantly changed the risk of hyper-LDL-cholesterolemia by haplotype at $P<0.05,{ }^{* *}$ at $P<0.01$, and ${ }^{* * *}$ at $P<0.0001$ 
white vegetables, mushrooms, fatty and white fish, seaweeds, fruits, and pickles (loading $\geq 0.4$ ). A high intake of the $\mathrm{BD}$ pattern had an interaction with haplotype $(P=$ 0.025). With low BD intake (the first tertile), hyper-LDLcholesterolemia risk was higher in the major allele and the heterozygote allele than minor allele whereas the concentrations decreased in the descending order of major, heterozygote, and minor alleles, in the participants with high BD intake (Fig. 2a). The Heterozygote group had higher serum LDL concentrations with low BD intake $(118.0 \pm 31.1 \mathrm{mg} / \mathrm{dL})$ than with the high $\mathrm{BD}$ intake (116.1 $\pm 30.2 \mathrm{mg} / \mathrm{dL}$; Fig. 2a). Participants with the Western diet pattern had diets high in bread, cake, cookies, and fast food, which explained $23.2 \%$ of the total variance of the participants, and those in the rice-based diet pattern consumed mainly rice to explain 18.0\% (Table 5). The Western diet pattern and mainly-rice diet pattern did not have interactions with haplotype and
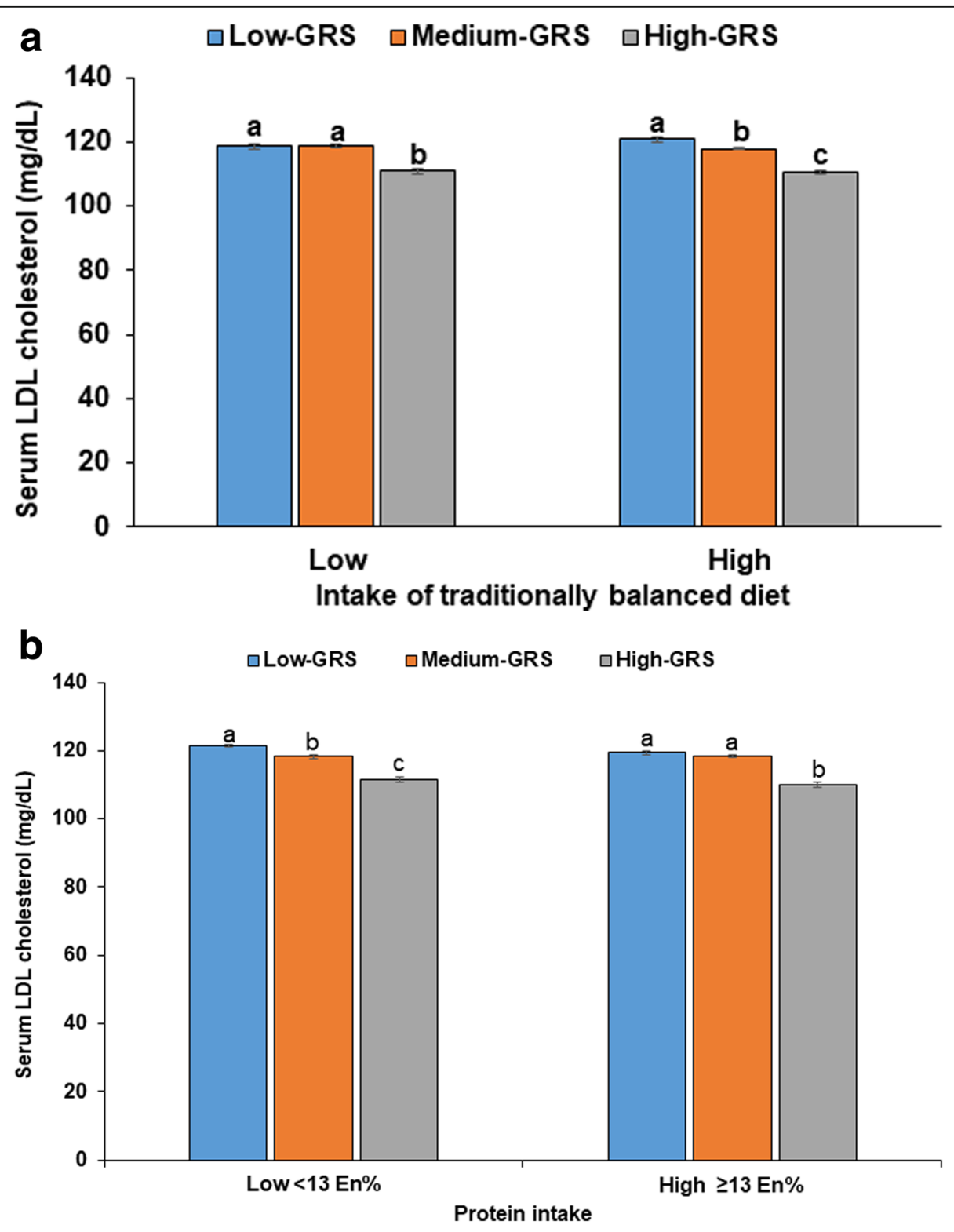

Fig. 2 Serum LDL concentrations of participants with major, heterozygotes and minor alleles of haplotype with SNPs in the 19q13 loci. A: Balanced diet pattern (cutoff value: 75th percentiles); B: Protein intake (cutoff value: 13 energy percent (En\%). Non-risk, heterozygotes, and risk alleles of each SNP were scored 0, 1, and 2, respectively, and the scores of each SNP in the haplotype were combined. The scores were divided into 3 categories (Major, Heterozygote, and Minor groups) by $0,1-2$, and 3-4, respectively. ${ }^{a, b, c}$ Means without a common letter differ among the different allele groups by Bonferroni test at $P<0.05$ in the low intake category. ${ }^{a^{\prime}, b^{\prime}, c^{\prime}}$ Means without a common letter differ among the different allele groups by Bonferroni test at $P<0.05$ in the high intake category 
Table 5 Adjusted odds ratio for the risk of low serum LDL concentrations by the haplotype ${ }^{a}$ after covariate adjustments according to the patterns of lifestyles

\begin{tabular}{|c|c|c|c|c|}
\hline & $\begin{array}{l}\text { Major } \\
(n=1782)\end{array}$ & $\begin{array}{l}\text { Heterozygote } \\
(n=1680)\end{array}$ & $\begin{array}{l}\text { Minor } \\
(n=301)\end{array}$ & $\begin{array}{l}\text { Gene-nutrient interaction } \\
P \text { value }\end{array}$ \\
\hline Low balanced diet ${ }^{b}$ & 1 & $0.994(0.869-1.136)$ & $0.722(0.570-0.914)$ & 0.0246 \\
\hline High balanced diet & & $0.854(0.769-0.948)$ & $0.640(0.540-0.759)$ & \\
\hline Low flour-rich diet ${ }^{c}$ & 1 & $0.876(0.795-0.965)$ & $0.540(0.456-0.639)$ & 0.6929 \\
\hline High flour-rich diet & & $0.881(0.825-0.940)$ & $0.596(0.534-0.666)$ & \\
\hline Low mainly-rice diet ${ }^{d}$ & 1 & $0.930(0.845-1.023)$ & $0.622(0.528-0.732)$ & 0.1788 \\
\hline High mainly-rice diet & & $0.858(0.804-0.917)$ & $0.560(0.501-0.626)$ & \\
\hline Low energy ${ }^{e}$ & 1 & $0.917(0.861 \sim 0.977)$ & $0.687(0.586 \sim 0.805)$ & 0.2870 \\
\hline High energy & & $0.918(0.799 \sim 1.056)$ & $0.680(0.529 \sim 0.873)$ & \\
\hline Low carbohydrate ${ }^{f}$ & 1 & $0.898(0.848 \sim 0.952)$ & $0.678(0.585 \sim 0.785)$ & 0.1922 \\
\hline High carbohydrate & & $0.931(0.765 \sim 1.134)$ & $0.718(0.515 \sim 1.002)$ & \\
\hline Low protein ${ }^{9}$ & 1 & $0.927(0.834 \sim 1.031)$ & $0.694(0.576 \sim 0.836)$ & 0.0059 \\
\hline High protein & & $0.933(0.870 \sim 0.999)$ & $0.671(0.544 \sim 0.815)$ & \\
\hline Low fat ${ }^{h}$ & 1 & $0.876(0.713 \sim 0.993)$ & $0.637(0.527 \sim 0.770)$ & 0.1690 \\
\hline High fat & & $1.034(0.929 \sim 1.151)$ & $0.734(0.606 \sim 0.888)$ & \\
\hline Low alcohol drinking & 1 & $0.898(0.851 \sim 0.947)$ & $0.645(0.554 \sim 0.751)$ & 0.3690 \\
\hline High alcohol drinking & & $0.910(0.765 \sim 1.084)$ & $0.868(0.649 \sim 1.161)$ & \\
\hline Non- + former smoking & 1 & $0.910(0.862-0.962)$ & $0.697(0.608-0.800)$ & 0.3436 \\
\hline Smoking & & $0.841(0.717-0.987)$ & $0.579(0.440-0.762)$ & \\
\hline Low coffee intake & 1 & $0.933(0.864 \sim 0.948)$ & $0.679(0.589 \sim 0.782)$ & 0.0935 \\
\hline High coffee intake & & $1.139(0.890 \sim 1.457)$ & $0.762(0.504 \sim 1.151)$ & \\
\hline Low physical activityk & 1 & $0.876(0.812 \sim 0.944)$ & $0.609(0.504 \sim 0.735)$ & 0.1105 \\
\hline High physical activity & & $0.887(0.698-1.127)$ & $0.738(0.610 \sim 0.970)$ & \\
\hline
\end{tabular}

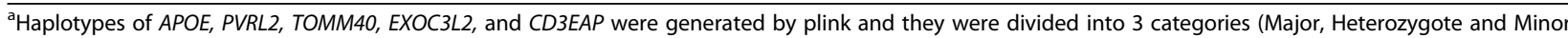
haplotype groups) by the alleles (0,1-2, and 3-4). Reference was the major haplotype allele

Values represent odds ratios and $95 \%$ confidence intervals

The cutoff points for dividing the values of each parameter into 2 groups were as follows: less than 75 th percentile of the respective diet intake ${ }^{b, c . d}$, less than

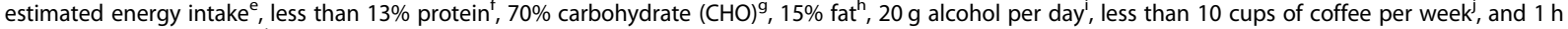
moderate activity per dayk. Multivariate regression models include the corresponding main effects, interaction terms of gene and main effects (energy and nutrient intake), and potential confounders such as BMI, residence area, gender, age, smoking, coffee, alcohol, physical activity and serum total cholesterol concentrations

with a low or high intake of the Western diet and mainly-rice diet patterns, hyper-LDL-cholesterolemia risk had a similar association with haplotype (Table 5).

Energy, carbohydrate, and fat intakes did not have interactions with the haplotype to influence hyper-LDLcholesterolemia. However, protein intakes had an interaction with the haplotype $(P=0.006)$. The serum LDL concentrations in the Major group with high protein intake $(119.4 \pm 31.9 \mathrm{mg} / \mathrm{dL})$ were lower than those in participants with low protein intake $(121.4 \pm 32.1 \mathrm{mg} / \mathrm{dL}$; Fig. 2b). Participants with the minor allele $(111.3 \pm 32.2$ $\mathrm{mg} / \mathrm{dL}$ ) had much lower LDL-cholesterol concentrations than those with the major allele $(120.7 \pm 32.0 \mathrm{mg} / \mathrm{dL})$.

\section{Discussion}

The $19 \mathrm{q} 13$ loci are important for modulating serum total-cholesterol and LDL concentrations in people of various ethnicities with family histories of type 2 diabetes [22]. In the current study, the haplotype comprised of 5 genetic variants in 19q13 had a strong association with hyper-LDL-cholesterolemia risk after adjusting for covariates. Hyper-LDL-cholesterolemia was associated with APOE rs7259620, PVRL2 rs403155, TOMM40 rs157581, EXOC3L2 rs10406604 and CD3EAP rs3212986 in the 19q13 loci. The haplotype included 5 selected SNPs that are associated with VLDL remnant delivery into the liver, conversion from VLDL to LDL in the blood, and LDL release into the circulation. HyperLDL-cholesterolemia risk was lower in the carriers of the minor alleles of the haplotype compared to carriers of the major allele, especially in those who had a high intake of the balanced diet pattern. The carriers with the minor allele had lower hyper-LDL-cholesterolemia risk compared to those with the major allele, especially in the high protein intake group. These results suggested that a personalized nutrition approach to the modulation 
of dietary patterns and nutrient intakes can reduce the risk of cardiovascular diseases and hyper-LDLcholesterolemia. This is the first study to show an interactive association between haplotype and nutrition intake for modulating LDL-cholesterol and cardiovascular disease risk.

LDL metabolism is closely associated with VLDL metabolism [11]. Thus, cholesterol and triglyceride in the circulation are interrelated and genetic factors may interact with dietary intake. The present study showed that participants having high serum LDL concentrations also had a high concentration of not only total cholesterol and triglyceride but also HDL. Since the increase of serum HDL concentrations were small, the ratio of LDL to HDL in the serum was much higher in the High-LDL than the Low-LDL. Furthermore, the incidence of cardiovascular diseases was much higher in the High-LDL than the Low-LDL even with the cutoff of $160 \mathrm{mg} / \mathrm{dL}$ serum LDL concentrations [23]. Thus, serum LDL concentrations need to be controlled with the modifications of lifestyles according to personal genetic variants.

Genetic variants that regulate serum LDL levels are reported to vary among ethnicities. $L D L R$ rs6511720 and rs57217136 are involved in enhancer-binding protein sites for a transcription factor for $L D L R$ and participants in this study who carried their minor alleles had increased $L D L R$ expression, which consequently reduces serum LDL concentrations [24]. G carriers of PCSK9 rs562556 had a lower risk of dyslipidemia including decreased serum LDL concentrations in meta-analysis without separating ethnicities [25]. Participants with the combination of ATP-binding cassette sub-family $G$ member-5 rs6720173-C, cholesterol 7 alpha-hydroxylase rs3808607-TT, and 7-dehydrocholesterol reductase rs760241-GG genotypes had lower serum LDL concentrations relative to those with opposite genotypes after a blended dairy consumption for 4 weeks ( 3 servings/day) [26]. The $A P O E-\varepsilon 4$ variant elevates serum LDL and homocysteine concentrations, thereby increasing the risk of cardiovascular diseases. Participants with CT and TT at methylenetetrahydrofolate reductase C667T are at higher risk of cardiovascular diseases than those with the CC genotype [27]. In the present study, genes in the 19q3 loci modulated serum LDL concentrations. The SNPs were APOE rs7259620, PVRL2 rs403155, TOMM40 rs157581, EXOC3L2 rs10406604, and CD3EAP rs3212986. In Han Chinese (784 with cardiovascular diseases and 730 without cardiovascular diseases) APOE rs7259620 had a positive association with cardiovascular diseases only in men, not women without adjusting for the covariates [28]. The study explained that the association may be due to gender differences in hormonal profiles, smoking status, alcohol-drinking, occupation, and dietary habits. These results are different from the present study in which APOE rs7259620 had a negative association with serum LDL concentration and cardiovascular disease incidence. Participants with minor allele had lower serum LDL concentrations and cardiovascular disease risk than those with major allele.

PVRL2 is an adherens junction protein. It responds to plasma cholesterol concentrations and it is involved in transendothelial migration of leukocytes to modulate vascular inflammation in PVRL2 deficient mice and presumably acts similarly in humans. Thus, PVRL2 is associated with the development of atherosclerosis in the endothelial sites [29]. TOMM40 is a protein embedded into the mitochondria outer membranes and is required for the movement of proteins into mitochondria. TOMM4O and APOE-TOMM4O loci are associated with dyslipidemia in Asians and non-Asians [30, 31]. EXOC3L2 is shown to interact with EXOC4 is a component of the exocyst complex. The complex is involved in exocytic vesicles of the cell membrane which mediate the process of exocytosis. It may be associated with LDLR exocytosis. It also regulates cell polarity and cell migration in endothelial cells. EXOC3L2 has not been previously reported to modulate dyslipidemia but EXOC3L2, APOE, TOMM40, and PVRL2 are reported to be Alzheimer's disease-susceptibility genes [32, 33]. CD3EAP encodes a nucleoprotein that is localized to the fibrillar centers of the nucleolus and it is mainly reported to be involved in lung cancer [34]. However, no previous studies have shown it to be related to dyslipidemia and cardiovascular diseases. Therefore, it was novel to demonstrate that the haplotype of $19 \mathrm{q} 3$ loci was shown to be related to the modulation of serum LDL concentrations in this study. The major alleles of the haplotype were a genetic risk for hyper-LDL-cholesterolemia.

Typically, circulating concentrations of LDL and triglyceride increase and decrease in parallel [35]. An unusual feature of these data was that the minor allele carriers at low risk of hypercholesterolemia were at elevated risk of hypertriglyceridemia. This may be due in part to the alleles decreasing biosynthesis of cholesterol in the liver while not affecting the availability of triglycerides derived from the diet. This would have the effect of increasing the content of triglyceride in VLDL and LDL particles relative to cholesterol. Genetic alterations that result in elevated triglycerides often involve variants that lower the activity of lipoprotein lipase, thereby impairing the removal of triglyceride from lipoprotein particles [35]. It may be possible that some of the genetic variants also affected triglyceride concentrations in such a manner. However, that cannot be determined from the current research data.

Overweight and obesity are risk factors for dyslipidemia [36]. Dietary components are also reported to modulate serum LDL concentrations [37]. The present 
study showed that energy, carbohydrates, fat intake did not have an interaction with the haplotype to modulate serum LDL concentrations. However, protein intake had an interaction with the haplotype. Carriers with minor alleles had lower hyper-LDL-cholesterolemia risk compared to those with major allele when consuming high amounts of foods in the balanced diet pattern as well as protein. However, the flour-rich diet and mainly-rice diet patterns did not have interactions with the haplotype. Other nutrients, coffee, and alcohol intake did not interact with haplotype to affect hyper-LDL-cholesterolemia risk.

\section{Strengths and limitations}

This study was the first study to investigate the haplotype in $19 \mathrm{q} 13$ loci that were associated with hyper-LDLcholesterolemia risk and the haplotype interacted with a balanced diet pattern and protein intake. In the haplotype of $19 \mathrm{q} 13$ loci, the major allele was the risk allele and a higher proportion of people had a genetic risk to have hyper-LDL-cholesterolemia, Genetic differences were associated with the events of cardiovascular diseases, especially myocardial infarction. These results can be used for the prevention of not only hyper-LDLcholesterolemia but also cardiovascular disease events. The balanced diet pattern and high protein intake protected against hyper-LDL-cholesterolemia in Major haplotype.

However, there were some limitations. 1) The data of this current study came from a cross-sectional setting even from a large hospital urban cohort. The results did not demonstrate cause and effect relationships. 2) Two SNPs included in the haplotype (rs10406604 and rs3212986) did not meet the conservative statistical significance $(P<5.0 \mathrm{E}-8)$. These SNPs might not be significantly associated with hyper-LDL-cholesterolemia. 3) Food intake was calculated from SQFFQ that was validated by 3-day food records [38]. The results might be somewhat under- and over-estimated for usual food intake. The fat intake was estimated but saturated, monounsaturated, and polyunsaturated fatty acid intake were not provided in KOGES although their fatty acid intake associated with hyper-LDL-cholesterolemia.

\section{Conclusion}

The present study suggested that the participants with hyper-LDL-cholesterolemia $(\geq 160 \mathrm{mg} / \mathrm{dL})$ were at higher risk of myocardial infarction and cardiovascular disease. Participants carrying the major allele haplotype in 19q13 loci had a higher risk of hyper-LDL-cholesterolemia by 1.5 times, compared to those carrying the major allele. The incidence of cardiovascular diseases, especially myocardial infarction, also had a positive association with the major allele of the haplotype. Participants carrying the major allele of the haplotype of $19 \mathrm{q} 13$ loci could reduce hyper-LDL-cholesterolemia and cardiovascular risk by consuming a balanced dietary pattern and with high protein intakes. Westernization of diet patterns can increase the susceptibility of hyper-LDL-cholesterolemia and cardiovascular diseases since the majority of people have risk alleles potentially in Asians including Korea. These results can be applied to precision nutrition protocols to prevent lowering serum LDL concentration and cardiovascular diseases and promote healthy aging.

\section{Supplementary information}

Supplementary information accompanies this paper at https://doi.org/10. 1186/s12944-020-01352-1.

Additional file 1. Supplemental Table 1 was provided in online only.

\section{Abbreviations}

LDL: Low-density lipoprotein cholesterol; HDL: High-density lipoprotein cholesterol; APOE: Apolipoprotein E; LDLR: LDL receptor; PCSK9: Proprotein convertase subtilisin-kexin type 9; SQFFQ: Semi-quantitative food frequency questionnaire; GWAS: Genome-wide association study; HWE: Hardy-Weinberg equilibrium; MAF: Minor allele frequency; BMI: Body mass index;

ANOVA: Analysis of variance; ORs: Odds ratios; Cl: 95\% confidence intervals; SNP: Single nucleotide polymorphism; PVRL2: Poliovirus receptor-related 2; TOMM40: Translocase of outer mitochondrial membrane 40;

EXOC3L2: Exocyst complex component 3-like 2; CD3EAP: CD3e molecule associated protein; LD: Linkage disequilibrium; VLDL: Very-low-density lipoprotein; Major: The major allele of the haplotype; Heterozygote: The heterozygote allele of haplotype; Minor: The minor allele of the haplotype; BD: A balanced diet pattern

\section{Authors' contributions}

SP formulated the research question, interpreted the data, and wrote the first draft of the manuscript. SK designed this study and analyzed the data. The authors read and approved the final draft of this manuscript to be published

\section{Funding}

This study was funded by a grant from the National Research Foundation of Korea (NRF) funded by the Ministry of Science and ICT (NRF-

2019R1A2C1007203).

\section{Availability of data and materials}

The datasets used and/or analyzed during the current study are available from the corresponding author on reasonable request.

\section{Ethics approval and consent to participate}

This study was approved by the Institutional Review Board of the Korean National Institute of Health for the KoGES (KBP-2015-055) and Hoseo University (1041231-150811-HR-034-01). The procedures were compliant with the Declaration of Helsinki and the ethical standards of the responsible committee on human experimentation. Written informed consent was obtained from all participants.

Consent for publication

Not applicable.

\section{Competing interests}

All authors declare no conflicts of interest.

Received: 25 May 2020 Accepted: 20 July 2020

Published online: 29 July 2020

\section{References}

1. Kim HJ, Kim Y, Cho Y, Jun B, Oh KW. Trends in the prevalence of major cardiovascular disease risk factors among Korean adults: results from the 
Korea National Health and nutrition examination survey, 1998-2012. Int Cardiol. 2014;174(1):64-72.

2. Kim YJ, Lee JS, Park J, Choi DS, Kim DM, Lee K, et al. Trends in socioeconomic inequalities in five major risk factors for cardiovascular disease in the Korean population: a cross-sectional study using data from the Korea National Health and nutrition examination survey, 2001-2014. BMJ Open. 2017;7(5):e014070.

3. Lee J, Son $\mathrm{H}$, Ryu OH. Management status of cardiovascular disease risk factors for dyslipidemia among Korean adults. Yonsei Med J. 2017;58(2):32638.

4. Lee SW, Kim HC, Lee HS, Suh I. Thirty-year trends in mortality from cardiovascular diseases in Korea. Korean Circulation j. 2015;45(3):202-9.

5. Rhee EJ, Kim HC, Kim JH, Lee EY, Kim BJ, Kim EM, et al. 2018 guidelines for the management of dyslipidemia. Korean J Intern Med. 2019;34(7):723-71.

6. Wade AT, Davis CR, Dyer KA, Hodgson JM, Woodman RJ, Murphy KJ. A Mediterranean diet supplemented with dairy foods improves markers of cardiovascular risk: results from the MedDairy randomized controlled trial. Am J Clin Nutr. 2018;108(6):1166-82.

7. Mertens E, Mullie P, Deforche B, Lefevre J, Charlier R, Huybrechts I, et al. Cross-sectional study on the relationship between the Mediterranean diet score and blood lipids. Nutr J. 2014;13:88.

8. Chiu S, Bergeron N, Williams PT, Bray GA, Sutherland B, Ronald M, et al. Comparison of the DASH (dietary approaches to stop hypertension) diet and a higher-fat DASH diet on blood pressure and lipids and lipoproteins: a randomized controlled trial. Am J Clin Nutr. 2016;103(2):341-7.

9. $\mathrm{Na} L$, Han $T$, Zhang $W$, Wu X, Na G, Du S, et al. A snack dietary pattern increases the risk of hypercholesterolemia in northern Chinese adults: a prospective cohort study. PLoS One. 2015;10(8):e0134294.

10. Lee J, Kim J. Association between dietary pattern and incidence of Cholesterolemia in Korean adults: the Korean genome and epidemiology study. Nutrients. 2018;10(1):53.

11. Kapourchali FR, Surendiran G, Goulet A, Moghadasian MH. The role of dietary cholesterol in lipoprotein metabolism and related metabolic abnormalities: a mini-review. Crit Rev Food Sci Nutr. 2016;56(14):2408-15.

12. Kathiresan S, Melander O, Guiducci C, Surti A, Burtt NP, Rieder MJ, et al. Six new loci associated with blood low-density lipoprotein cholesterol, highdensity lipoprotein cholesterol or triglycerides in humans. Nat Genet. 2008; 40(2):189-97.

13. Subramanian S, Gottschalk WK, Kim SY, Roses AD, Chiba-Falek O. The effects of PPARgamma on the regulation of the TOMM40-APOE-C1 genes cluster. Biochim Biophys Acta Mol basis Dis. 2017;1863(3):810-6.

14. Robles-Osorio L, Huerta-Zepeda A, Ordóñez ML, Canizales-Quinteros S, DíazVillaseñor A, Gutiérrez-Aguilar R, et al. Genetic heterogeneity of autosomal dominant hypercholesterolemia in Mexico. Arch Med Res. 2006;37(1):102-8.

15. Zhang QS, Browning BL, Browning SR. Genome-wide haplotypic testing in a Finnish cohort identifies a novel association with low-density lipoprotein cholesterol. Eur J Hum Genet. 2015;23(6):672-7.

16. Pirim D, Radwan ZH, Wang X, Niemsiri V, Hokanson JE, Hamman RF, et al. Apolipoprotein E-C1-C4-C2 gene cluster region and inter-individual variation in plasma lipoprotein levels: a comprehensive genetic association study in two ethnic groups. PLoS One. 2019;14:e0214060.

17. Kim Y, Han BG. Cohort profile: the Korean genome and epidemiology study (KoGES) consortium. Int J Epidemiol. 2017:46(11):1350.

18. Park S, Ahn J, Lee BK. Very-low-fat diets may be associated with an increased risk of metabolic syndrome in the adult population. Clin Nutr. 2016;35(5):1159-67.

19. Friedewald WT, Levy RI, Fredrickson DS. Estimation of the concentration of low-density lipoprotein cholesterol in plasma, without use of the preparative ultracentrifuge. Clin Chem. 1972;18(5):499-502.

20. DRI Committee in Korea. 2015 dietary reference intakes for Koreans 2015. Seoul: Ministry of Health and Welfare; 2016.

21. Barrett JC, Fry B, Maller J, Daly MJ. Haploview: analysis and visualization of LD and haplotype maps. Bioinformatics. 2005;21(6):263-5.

22. Paththinige CS, Sirisena ND, Dissanayake V. Genetic determinants of inherited susceptibility to hypercholesterolemia - a comprehensive literature review. Lipids Health Dis. 2017;16(1):103.

23. LaRosa JC. Reduction of serum LDL-C levels: a relationship to clinical benefits. Am J Cardiovasc Drugs. 2003;3(4):271-81.

24. Fairoozy RH, White J, Palmen J, Kalea AZ, Humphries SE. Identification of the functional variant(s) that explain the low-density lipoprotein receptor (LDLR)
GWAS SNP rs6511720 association with lower LDL-C and risk of CHD. PLoS One. 2016:11(12):e0167676.

25. Chuan J, Qian Z, Zhang Y, Tong R, Peng M. The association of the PCSK9 rs562556 polymorphism with serum lipids level: a meta-analysis. Lipids Health Dis. 2019;18(1):105.

26. Abdullah MMH, Eck PK, Couture P, Lamarche B, Jones PJH. The combination of single nucleotide polymorphisms rs6720173 (ABCG5), rs3808607 (CYP7A1), and rs760241 (DHCR7) is associated with differing serum cholesterol responses to dairy consumption. Appl Physiol Nutr Metab. 2018; 43(10):1090-3.

27. Long Y, Zhao XT, Liu C, Sun YY, Ma YT, Liu XY, et al. A case-control study of the Association of the Polymorphisms of MTHFR and APOE with risk factors and the severity of coronary artery disease. Cardiology. 2019;142(3):149-57.

28. Wu N, Liu G, Huang Y, Liao Q, Han L, Ye H, et al. Study of the association of 17 lipid-related gene polymorphisms with coronary heart disease. Anatol J Cardiol. 2018;19(6):360-7.

29. Rossignoli A, Shang MM, Gladh H, Moessinger C, Asl HF, Talukdar HA, et al. Poliovirus receptor-related 2: a cholesterol-responsive gene affecting atherosclerosis development by modulating leukocyte migration. Arterioscler Thromb Vasc Biol. 2017:37(3):534-42.

30. Kong X, Zhao Q, Xing X, Zhang B, Zhang X, Hong J, et al. Genetic variants associated with lipid profiles in Chinese patients with type 2 diabetes. PLoS One. 2015;10(8):e0135145.

31. Talmud PJ, Drenos F, Shah S, Shah T, Palmen J, Verzilli C, et al. Gene-centric association signals for lipids and apolipoproteins identified via the HumanCVD BeadChip. Am J Hum Genet. 2009;85(5):628-42.

32. Wu QJ, Sun SY, Yan CJ, Cheng ZC, Yang MF, Li ZF, et al. EXOC3L2 rs597668 variant contributes to Alzheimer's disease susceptibility in the Asian population. Oncotarget. 2017:8(12):20086-91.

33. Lutz MW, Casanova R, Saldana S, Kuchibhatla M, Plassman BL, Hayden KM. Analysis of pleiotropic genetic effects on cognitive impairment, systemic inflammation, and plasma lipids in the health and retirement study. Neurobiol Aging. 2019;80:173-86.

34. Yin J, Wang H, Vogel U, Wang C, Hou W, Ma Y. Association and interaction of NFKB1 rs28362491 insertion/deletion ATTG polymorphism and PPP1R13L and CD3EAP related to lung cancer risk in a Chinese population. Tumour Biol. 2016;37(4):5467-73.

35. Dron JS, Hegele RA. Genetics of triglycerides and the risk of atherosclerosis. Curr Atheroscler Rep. 2017;19(1):31.

36. Siri-Tarino PW, Krauss RM. Diet, lipids, and cardiovascular disease. Curr Opin Lipidol. 2016;27(4):323-8.

37. Kopin L, Lowenstein C. Dyslipidemia. Ann Intern Med. 2017;167(11):itc81-96.

38. Zhou JY, Song MY, Park S. Carbohydrate and sodium intake and physical activity interact with genetic risk scores of four genetic variants mainly related to lipid metabolism to modulate metabolic syndrome risk in Korean middle-aged adults. Brit J Nutr. 2019;122(8):919-27.

\section{Publisher's Note}

Springer Nature remains neutral with regard to jurisdictional claims in published maps and institutional affiliations.

Ready to submit your research? Choose BMC and benefit from:

- fast, convenient online submission

- thorough peer review by experienced researchers in your field

- rapid publication on acceptance

- support for research data, including large and complex data types

- gold Open Access which fosters wider collaboration and increased citations

- maximum visibility for your research: over $100 \mathrm{M}$ website views per year

At $\mathrm{BMC}$, research is always in progress.

Learn more biomedcentral.com/submission 